Check for updates

Cite this: Analyst, 2021, 146, 196

\title{
Portable, low cost and sensitive cavity enhanced absorption (CEA) detection $\uparrow$
}

\author{
Andrew Teggert, (DD a Harish Datta, ${ }^{a, b}$ Stephen Mclntosh, ${ }^{c}$ Barry Warden, (DD c \\ Simon Bateson, ${ }^{d}$ Fathi Abugchem (D) ${ }^{d}$ and Zulfiqur Ali $\mathbb{D} * d$
}

\begin{abstract}
Absorption is a widely used technique for a range of different applications. It has lower sensitivity than many other techniques such as fluorescence which has 100 to 1000 times higher sensitivity than absorption. Optical cavity approaches have been developed where the light passes back and forth, within the sample, between two high reflectivity mirrors to increase the pathlength and sensitivity. These approaches have not yet, however, been widely used for analytical applications and for point-of-care diagnostics. Here we show a portable cavity enhanced absorption (CEA) spectrometer and a low cost point-of-care (POC) reader with $C E A$ detection with mechanical elements fabricated using 3D printing. The CEA spectrometer can be used in both single pass and multi-pass cavity enhanced mode to provide measurements in the visible region that are very sensitive and over a wide dynamic range. The CEA mode was shown for Rhodamine B dye to increase the pathlength 57.8 fold over single pass measurements and an LOD of $7.1 \times 10^{-11} \mathrm{M}$. The cost of the CEA POC reader was reduced by use of narrow band LEDs, photodiodes and removal of fibre optic coupling and with a 14 fold increase in the pathlength over conventional single pass microplate readers. The CEA POC reader was demonstrated for immunoassay of C-Reactive Protein (CRP), Procalcitonin (PCT) and Interleukin 6 (IL-6), towards a three biomarker panel to aid the diagnosis of sepsis. The CEA POC reader can be integrated with wireless connectivity for cloud based data sharing. We show here the potential for the wider use of optical cavity approaches where there is a need for sensitive absorption measurements and also for low cost point-of-care diagnostics.
\end{abstract}

Received 16th September 2020 Accepted 23rd October 2020

DOI: 10.1039/d0an01852j

rsc.li/analyst length. In the presence of a sample and with low background noise then the amplification on the detector can be increased to the extent that single molecule detection can be achieved. For absorption measurements, the intensity of light in the absence of sample - which is typically large - is measured against a small change of absorption that arises with a sample. The sensitivity is therefore limited by the ability to measure a small change in absorption of the sample from a large background absorption.

It is known from the Beer-Lambert law that the absorbance of a sample will dependent on the molar absorption coefficient $(\varepsilon)$, the concentration $(C)$ and the pathlength $(l)$. The molar absorption coefficient is a fundamental property of the analyte molecule and the concentration will be characteristic of the sample being measured. An increase in the sensitivity of absorbance measurements for a particular sample can therefore be achieved by physically increasing the pathlength or ensuring multiple passes of light through the sample by using multipass absorption cells such as the White and Herriott cells. These approaches are, however, limited by the number of passes that can be achieved and often require relatively large sample volumes. An alternative approach is to place the sample between an optical resonator, within which light is introduced, with the objective of increasing the effective path- 
length to magnify the optical absorption effect and increase the sensitivity. ${ }^{1,2}$ The resonator can take different forms including an optical fibre loop or two highly reflective mirrors which are typically formed using dielectric materials. Reflection of light from each mirror will result in a small amount of light being lost because of the limitation of the mirror reflectivity; typically this can be $1 \%$ or less, in addition to absorption and scattering losses within the cell.

In Cavity Ring Down Spectroscopy (CRDS) a brief high intensity pulse of light is introduced and the leakage of light from the back of one high reflectivity mirror is measured. The loss of intensity of light from absorption or scattering gives rise to an exponential decay in the signal. ${ }^{3}$ The time constant of this decay is known as the ring down time and depends on the mirror reflectivity and optical losses. If the mirror reflectivity stays the same then the change in the ring-down time can be associated with a change in the absorption or scattering within the sample. In CRDS, the rate of decay is determined and so the measurement is independent of the light source power fluctuations and the measurements can be very sensitive. Also, the absorption coefficient of the sample can be measured if the reflectivity of the mirror is known. A drawback of CRDS has been that the ring down time takes place over micro to nanosecond timescales and has required fast and so far generally expensive detection equipment. Vallance et.al., used a Nd/Yag laser and PMT detection with display on a digital oscilloscope for coupling CRDS with a microfluidic chip. ${ }^{4}$ Kaminski et.al., used a supercontinuum light source and avalanche photodiode array for absorption measurements in liquids with CRDS. ${ }^{5}$ In a different approach, Girkin et.al., have used intracavity laser absorption spectroscopy (ICLAS) for detection of chemicals in solution with the enhanced absorption arising from multiple passing through the sample and a balance between the laser gain and the intracavity absorption. ${ }^{6}$

In an alternative Cavity Enhanced Absorption (CEA) approach, instead of measuring the ring down time the total intensity of light leaking out of the back of the mirror is measured. ${ }^{7}$ This has the advantage of allowing slower and cheaper detection and is much more akin to conventional absorption spectrometry where the intensity is measured in the presence and absence of the sample. Enhancements of this approach have included use of a high intensity lamp ${ }^{8}$ as a broadband light source instead of a laser to provide measurements across a range of wavelengths and alternatively a high intensity $\mathrm{LED}^{9}$ as a light source to reduce the cost. A drawback to CEA measurements has been that it is not possible to theoretically determine the number of reflections that are taking place within the optical cavity, so calibration is required to determine the absorption coefficient of analytes. CEA measurements have largely been used within gas phase studies, with relatively few studies in the liquid phase ${ }^{10,11}$ since there are much higher losses that arise from scattering and absorption of liquids. Liquid molecules will have higher level of scattering since there will inherently be a higher concentration over gas molecules.

The use of CEA measurements within liquids is very attractive since there are a wide range of applications to which the technique can be applied. Initial studies in a 2-mm cuvette with a wide range of samples at visible wavelengths showed a $\sim 50$-fold improvement in sensitivity compared to conventional UV-visible spectroscopy using a visible LED as a light source. ${ }^{12}$ CEA has been used as a detector for High Performance Liquid Chromatography (HPLC) measurements by placing a HPLC flow cell $(70 \mu \mathrm{L})$ into an optical cavity. A 50-70-fold improved sensitivity was demonstrated over a conventional HPLC instrument. ${ }^{13}$ The higher sensitivity for CEA detection has been used within liquid-phase stopped flow kinetics for the measurement of fast reactions through slowing down the reaction rate by use of lower concentration of reagents. ${ }^{14}$ Vallance et.al., used a very interesting approach of supercontinuum radiation with broadband external mirrors for absorbance measurements within microlitre volume aqueous phase droplets in a microfluidic chip assembly. ${ }^{15}$ Morgan et.al., demonstrated an exciting approach of a disposable microfluidic chip with integrated cavity mirrors using enhanced specular reflector II film achieving $\times 5$ sensitivity enhancement allowing micromolar range detection for a $50 \mu \mathrm{m}$ optical pathlength. ${ }^{16}$

CEA detection therefore offers the potential for approaching the sensitivity of fluorescence but with the advantages of lower cost due to fewer optical elements and not necessarily requiring a label. For example; Enzyme-linked immunosorbent assays (ELISAs) are widely used for clinical analysis. CEA detection has shown a 39 fold increase in sensitivity for a 96 well microtiter plate commercially available osteocalcin ELISA as well as a 115-fold increase in sensitivity for a commonly used ELISA colorimetric reaction of streptavidin-horseradish peroxidase (STREP-HRP) with Tetramethylbenzidine (TMB). ${ }^{17}$ The setup included: motorised XY stage, with stepper motor controller unit, placed between the cavity mirrors using four translating post holders for fine adjustment of tilt relative to the cavity mirrors; Andor spectrograph coupled to thermoelectrically cooled CCD camera; and optical elements comprising lenses and irises to collimate the divergent output. There is a need to reduce the overall cost and size of component elements for wider applicability. We show here CEA detection for a portable horizontal cavity spectrometer and a low cost vertical cavity point-of-care (POC) diagnostic reader, fabricated using a combination of $3 \mathrm{D}$ printing and machined parts, for immunoassay of CRP, PCT and IL-6 as three well established biomarkers for sepsis.

\section{Experimental}

\section{CEA spectrometer}

The developed visible CEA spectrometer is shown in Fig. 1 and comprises a horizontal optical cavity with LED light source, mirrors and a microspectrometer for detection. The structural elements of the CEA spectrometer were fabricated using inket 3D printing (i3DP). The CEA spectrometer is controlled by a Raspberry Pi microcomputer and a software coded using C++ programming language and Qt5. A graphical user interface (GUI) was developed for control of all of the CEA spectrometer 

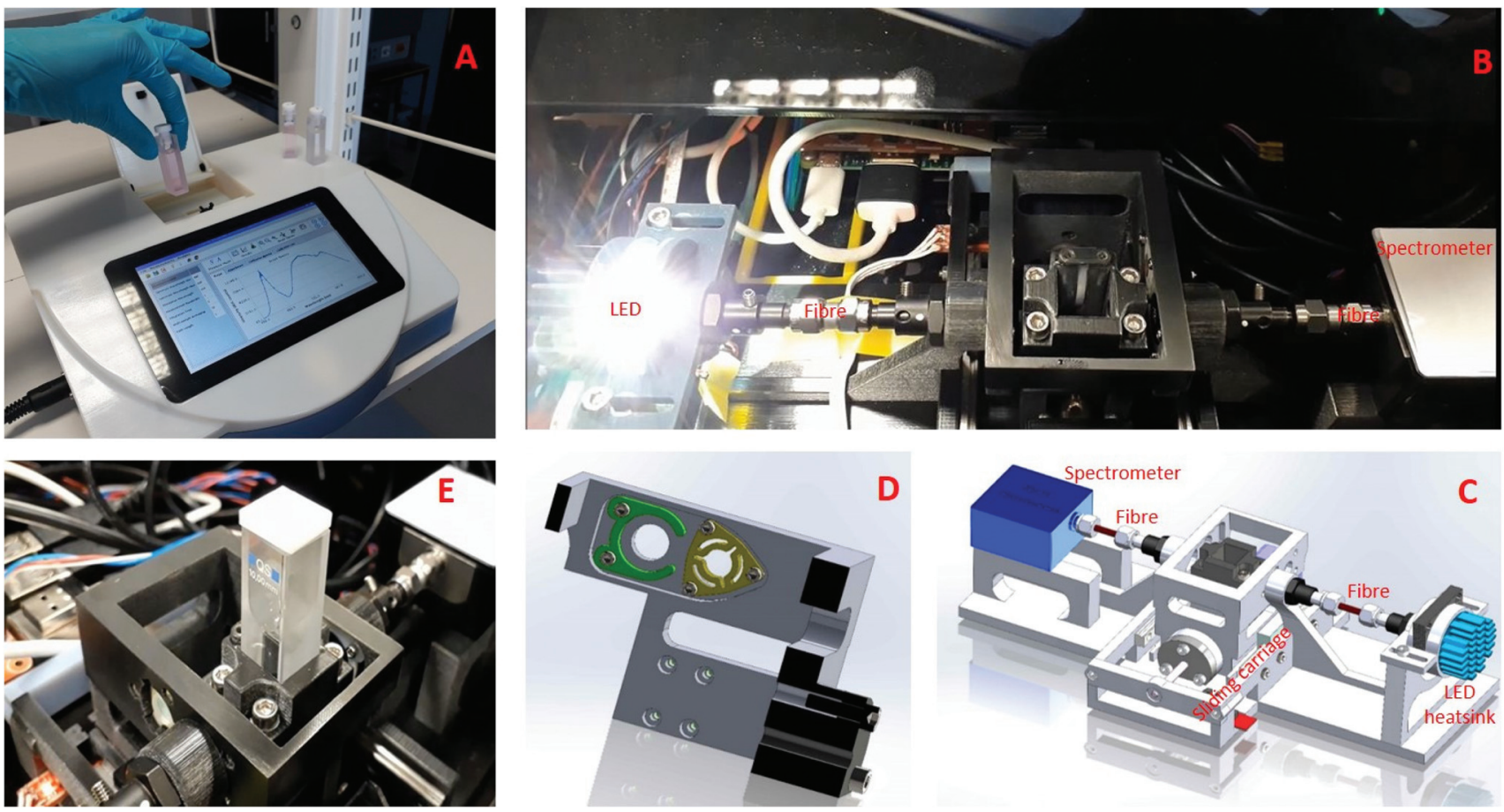

Fig. 1 (a) CEA spectrometer with $1 \mathrm{~cm}$ cuvette to show scale with structural elements fabricated using i3DP (b and c) horizontal optical cavity showing the led, fibre coupling, sample holder and sliding carriage (d) custom holder with standard filter (green) or mirror (yellow) as part of the sliding carriage for single and multi-pass measurements (e) sliding carriage with custom holder assembly.

functions as well as acquisition of data. The broadband high power white LED (Luxeonstar, cool white $6500 \mathrm{~K}$ ) was attached to a LED heat sink (Farnell) to avoid overheating. The light source is collimated using a collimating lens (Ocean Optics, 74-UV, fused silica, focal length $10 \mathrm{~mm}$ and focal length 200-2000 nm) on the input and output of a multimode optical fibre with the light being input into an optical cavity which was formed by two fused silica plano-concave high reflectivity mirrors, $99 \pm 0.5 \%$ dielectric mirrors (diameter $6.35 .0-0.1 \mathrm{~mm}$, thickness $4 \pm 0.1 \mathrm{~mm}, 420-640 \mathrm{~nm}$ ) with a radius of curvature of $38.0 \mathrm{~mm}$. The light exiting the output mirror is coupled using a collimating lens at the input of the multimode optic fibre and then to a microspectrometer (Ocean Optics, STS-UV, size $40 \mathrm{~mm} \times 42 \mathrm{~mm} \times 24 \mathrm{~mm}$ ), allowing measurement to be made in a wavelength range of $190-650 \mathrm{~nm}$. The mirrors were press fit into custom holders within a sliding carriage with a stepper motor to move the sliding carriage (Fig. 1D and E). Light was therefore able to pass from the optical fibre through either a standard (filter holder, green) or CEA channel (mirror holder, yellow) allowing both conventional single pass and highly sensitive CEA measurements to be made.

\section{CEA point-of-care (POC) reader}

A simpler, fixed wavelength aimed at POC is shown in Fig. 3 and comprised: an optical reading head; a moving tray; and two high power narrow band LEDs, in the $450 \mathrm{~nm}$ and $540 \mathrm{~nm}$ regions, with dual detectors sensitive at these wavelengths. The optical reading head holds the optical cavity with the LED assembly, sensing assemblies and the adjustment stages mounted to a rigid arm (Fig. 3C and inset). The LEDs are aligned to the centre of the mirrors and therefore the centre of the sample well that is being read. A cube beam splitter was used to allow both LEDs to illuminate along the same optical axis and through the centre of the well. The performance of the CEA POC reader were initially investigated using Tartrazine as an absorption standard, since it absorbs light at $428 \mathrm{~nm}$ which is close to the immunoassay measurements at $450 \mathrm{~nm}$, over a range of concentrations from $2 \times 10^{-7}-2 \times 10^{-5} \mathrm{M}$ of Tartrazine (Sigma-Aldrich T0388) in deionised water. Conventional single pass measurements were performed using EL800 (Biotek) and LT-4000 (EuroClone) microplate readers.

\section{Sepsis biomarker immunoassay}

The sepsis biomarker immunoassays were implemented in a custom microwell plate, shown Fig. 3D and comprised: polylysine coated glass slide as the bottom layer; an inkjet 3D printed middle layer to create the microwell; and a uncoated glass slide that was placed on the middle layer to form the lid. The ELISA protocol was adjusted to suit the smaller $165 \mu \mathrm{L}$ total volume for each of the microwells. The general scheme used for the sepsis biomarker ELISAs incorporated within microwell plate for the CEA POC reader is shown in Fig. 5A. For the CRP immunoassay, CRP capture antibodies (R\&D Systems, Abingdon, UK) are immobilised onto the bottom layer polylysine coated glass slide. This is achieved by coating each well with an antibody solution prepared by diluting the lyophilised antibodies to $10 \mu \mathrm{g} \mathrm{mL}^{-1}$ in phosphate buffered saline (PBS) and incubating for four hours at room tempera- 

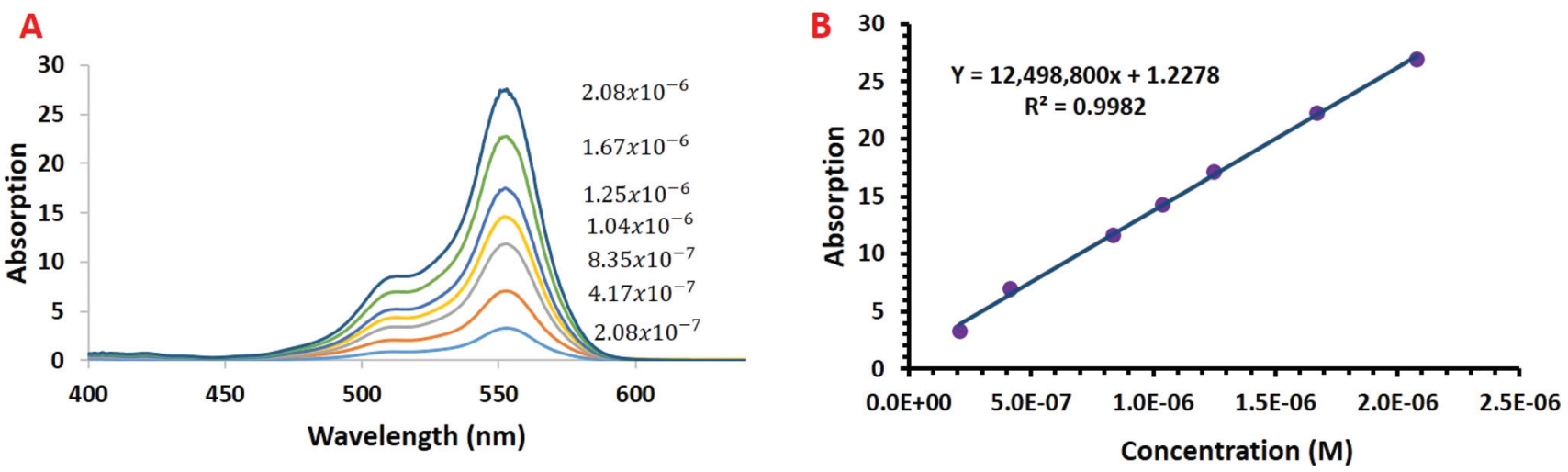

Fig. 2 (a) Cavity enhanced absorption spectrum for rhodamine b and (b) calibration graph of absorption versus rhodamine b concentration with the CEA spectrometer.
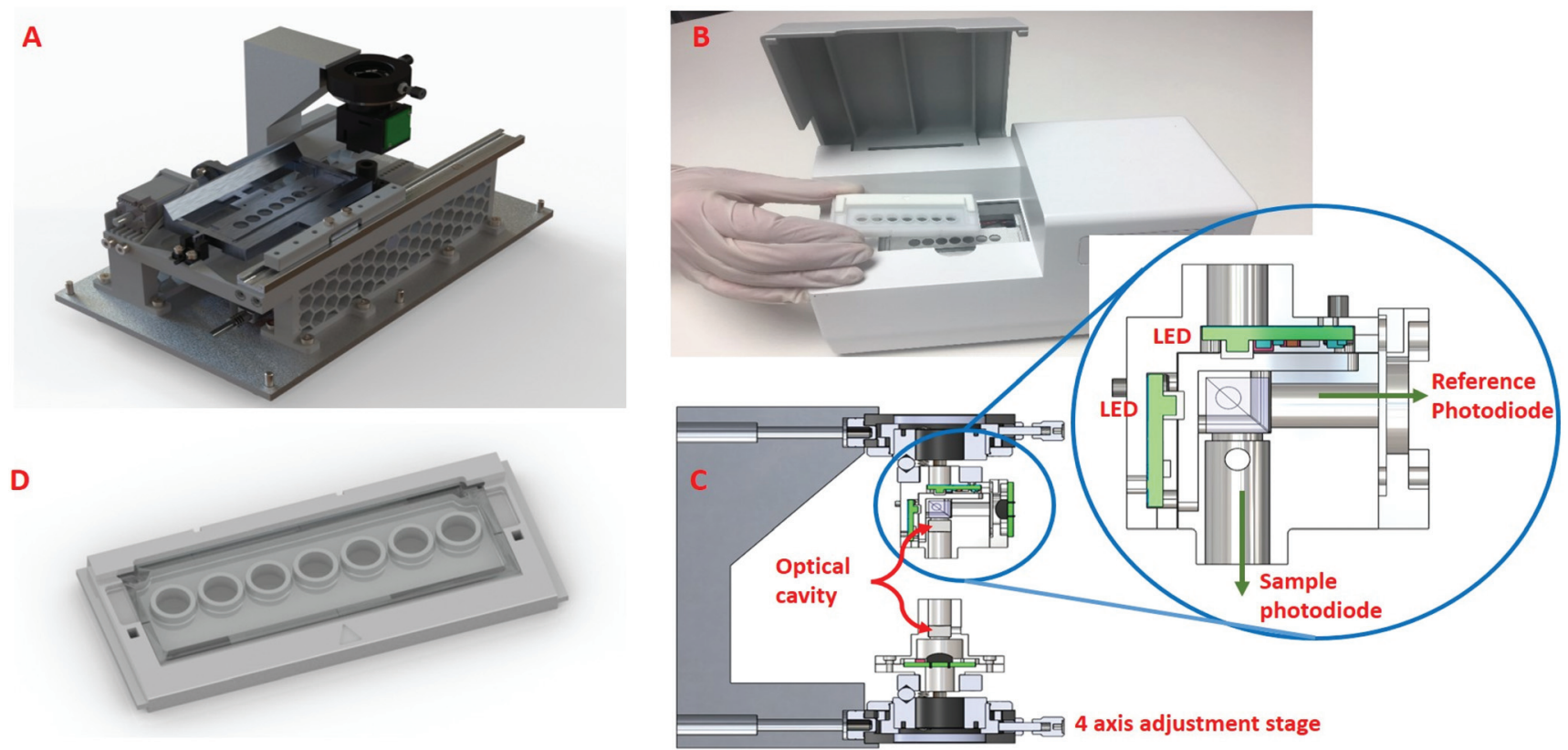

Fig. 3 (a) CEA POC reader assembly without the external enclosure (b) CEA POC reader with external enclosure and microwell plate for incorporating immunoassay (c) optical reading head assembly showing the optical cavity formed by two highly reflective mirrors which are precisely aligned to each other using two identical 4 axis adjustment stages mounted on a rigid arm as well as the led assembly and the reference and sample photodiodes (d) microwell plate consumable with polylysine coated glass slide as bottom and a glass slide for the lid.

ture. After incubation, the antibody solution is aspirated and the wells washed with a wash buffer consisting of $0.05 \%$ Tween in PBS. A blocking solution of $1 \%$ bovine serum albumin in PBS is then added to each well and incubated for one hour at room temperature to reduce the amount of non-specific protein binding, followed by a further wash. Each sample cartridge has five wells available for analysis. Sample is then added to the sample wells, incubated and washed. A biotinylated CRP detection antibody solution prepared by diluting the lyophilised antibodies to $90 \mathrm{ng} \mathrm{mL}^{-1}$ in $1 \%$ bovine serum albumin in PBS is added, incubated and washed, followed by addition of streptavidin-horseradish peroxidase which is again incubated and washed. The final step is the addition of TMB substrate to give a blue coloured product followed by sulphuric acid $(2 \mathrm{~N})$ which quenches the reaction and forms a yellow colour, the absorbance of which can be measured at $450 \mathrm{~nm}$. A similar approach was used for the development of PCT and IL-6 immunoassay.

\section{Results and discussion}

\section{Low cost and portable visible CEA spectrometer}

The CEA spectrometer comprises both a standard channel and a cavity enhanced channel to allow measurements to be performed over a wide dynamic range with high sensitivity in the visible region. This also allowed convenient measurement of 
the cavity enhancement. The high sensitivity of the system arising from the increased effective pathlength allows the measurement of the optical density of reagents at very low concentrations, below those detectable with standard spectrophotometers.

In case of a mirror reflectivity of $R \geq 0.99,99 \%$ or more of the incident light on the mirrors is reflected. An ideal mirror without absorption or scattering losses will have a maximum transmission of $T=1-R$, that is $\sim 1 \%$ of the incident intensity enters the cavity through the back of the first mirror. The separation distance $(L)$ between the cavity mirrors is determined by both the radius of the curvature of the mirrors $\left(r_{\mathrm{oc}}\right)$ and the selected confocal cavity configuration. The mirrors in this stable configuration produce an average beam diameter of 5-6 $\mathrm{mm}$ along its length, well suited for cuvette based measurements. It is important to ensure that the sample holder is perfectly parallel to the high reflectivity mirrors.

The mechanical elements of the prototype CEA spectrometer (Fig. 1) was fabricated using inkjet 3d printing (i3DP) which provides a convenient means of rapidly prototyping the mechanical and structural elements of the CEA spectrometer. To allow a wide dynamic range of absorbance measurements the CEA spectrometer was developed to allow both single pass and CEA measurements. There was therefore a need for the high reflectivity mirrors to be able to be moved in and out of the light path as required. The alignment of the mirrors to each other and the mirrors alignment to cuvette holder is fundamentally important for the performance of the CEA spectrometer. Alignment of the mirrors was carried out manually by adjusting the three screws in the holders and this obviated the need for an automated system for mirror alignment which would be expensive. It was found that once the mirrors were set they did not need to be regularly realigned. A program was added to the CEA software to correct any horizontal misalignment that may occur due to the horizontal movement of the mirrors to allow both single pass and CEA measurements. The CEA software records the raw data from the spectrometer in intensity mode, converts this into absorbance and then performs the other necessary calculations. The data and the results of the calculations are plotted and displayed on the GUI and also saved in files in the Raspberry Pi. The GUI is also used to set up and control all of the functions of the CEA which includes: selection of single pass or CEA measurements, spectrum wavelength; peak absorption wavelength of analyte; measurement bandwidth; integration time; multi-sample averaging and extinction coefficient.

Previous gas phase ${ }^{18}$ studies have shown that the absorption for CEA measurements can be expressed as:

$$
\begin{gathered}
\left(\frac{I_{0}(\lambda)}{I(\lambda)}\right)_{\text {cavity }}=1+\frac{\alpha l}{(1-R)} \\
\alpha=2.303 \varepsilon_{\lambda} C
\end{gathered}
$$

where $I_{0}(\lambda)$ and $I(\lambda)$ are the transmitted intensities in the presence and absence of the absorber in the cavity, $l$ is the optical path length through the sample in the cavity in $\mathrm{cm}, R$ is the average wavelength dependent mirror reflectivity, $\varepsilon_{\lambda}$ is the wavelength dependent molar extinction coefficient in $\mathbf{M}^{-1}$ $\mathrm{cm}^{-1}$, and $C$ is the concentration of the sample in $\mathrm{M}$. There is therefore a linear relationship between $I_{0}(\lambda) / I(\lambda)$ and the concentration of the analyte. The enhancement over conventional single pass measurements is therefore given by the term $1 /(1-R)$ and is termed as the Cavity Enhancement Factor (CEF). Rearranging eqn (1) in terms of $\mathrm{CEF}$ and combining with eqn (2) yields:

$$
\operatorname{CEF}(\lambda)=\frac{\left(\frac{I_{\mathrm{o}}(\lambda)}{I(\lambda)}-1\right)_{\text {cavity }}}{2.303 \varepsilon_{\lambda} C l}
$$

Using an approach that has been described previously, ${ }^{17}$ the CEF at a particular wavelength can be determined if the cavity absorption (numerator term) is known along with the concentration of analyte, molar extinction coefficient at a given wavelength $\varepsilon_{\lambda}$ and the base pathlength of measurement. Alternatively, the CEF values can be averaged over a range of concentrations to provide a more representative value. Eqn (3) can be arranged to the form of eqn (4).

$$
\left(\frac{I_{0}(\lambda)}{I(\lambda)}-1\right)_{\text {cavity }}=\operatorname{CEF}(\lambda) 2.303 \varepsilon_{\lambda} C l
$$

it can be seen that a plot of cavity absorption versus concentration would give a cavity absorption gradient $\left(\mathrm{CA}_{\text {gradient }}\right)$ which would allow determination of the CEF from eqn (5) by either substituting in values of $\varepsilon$ and $l$ or using the gradient of a plot of single pass absorbance versus concentration ( $\left.\mathrm{SP}_{\text {gradient }}\right)$.

$$
\mathrm{CEF}=\frac{\mathrm{CA}_{\text {gradient }}}{2.303 \varepsilon l}=\frac{\mathrm{CA}_{\text {gradient }}}{2.303 \mathrm{SP}_{\text {gradient }}}
$$

The measurement process for both the CEA and single pass measurements are very similar, except in the case of the former where the sample is located within the optical cavity using the sliding carriage arrangement. The data for CEA measurements needs to be converted from absorbance to absorption $\left(10^{\text {absorbance }}-1\right)$. For measurement, there is a need to first maximise the light intensity reaching the detector. Dark and Reference (zero absorbance) measurements are made with an empty optical cavity, and no cuvette present in the sample holder. Dark measurements are made with a shutter in front of the optical cavity. For reference measurements, the shutter is removed and light passes through the empty optical cavity. The intensity spectra for the empty cavity are recorded and an intensity count of around 14 000-14 500 was found to be optimum for an empty cavity. A low intensity count or oversaturation of the detector can be adjusted by changing the integration time to maximise the light intensity in the empty cavity, the integration time being the length of time for the detector to collect photons before passing the accumulated charge to the analogue to digital convertor for processing. Although a higher integration time allows more light to be collected it may also result in more electrical noise. A default integration time of 5 milliseconds was used for 
single pass measurements and 100 milliseconds for the CEA measurements. Insufficient intensity counts indicate misaligned light source or mirrors, requiring adjustment.

The recorded absorbance data without the sample present is used to calculate the noise, or $\Delta \mathrm{ABS}_{\text {min }}$, by measuring the standard deviation in absorbance around the wavelength of interest which is ordinarily the wavelength of peak cavity absorption. Intensity spectra were recorded over six wavelengths - around the peak analyte absorbance wavelength with 10 repeat measurements and these are paired with one being treated as $I_{0}$ and the other as $I$ resulting in five absorbance spectra. A standard deviation is calculated for the absorbances over the six wavelengths and this is then averaged for the five absorbance spectra to give the $\Delta \mathrm{ABS}_{\min }$ as in eqn (6)

$$
\Delta \mathrm{ABS}_{\min }=\operatorname{AVR}\left\{\operatorname{SD}\left(\log _{10} \frac{\left[I_{0}-\text { dark }\right]}{[I-\text { dark }]}\right)\right\}
$$

The resulting $\triangle \mathrm{ABS}_{\min }$ is normalised to a total acquisition time $(t)$ of one second (eqn (7)) to allow a fair comparison and represents the minimum detectable absorbance changed in the cavity absorption.

$$
\Delta \mathrm{ABS}_{\min }(t)=\Delta \mathrm{ABS}_{\min } \times \sqrt{ } t
$$

A cuvette with a blank solvent solution is placed into the optical cavity and the intensity spectra is recorded and this is used to calculate the losses arising from the cuvette. The integration time can be adjusted to maximise the light intensity without oversaturating the detector. Dark and reference measurements are made with the blank solvent solution and the intensity spectra are saved within memory to serve as the $I_{0}$ (intensity of light in the absence of sample). The blank solution is replaced with standard samples at different concentrations with any replicate measurements. The intensity spectra for a sample $(I)$ at a specific concentration are recorded and used with the stored blank solution intensity for determination of either the sample absorbance or absorption for respectively single pass and CEA measurements. A mean absorbance or absorption is calculated where the same sample concentration was resampled. It should be noted that baseline drift can occur and therefore the GUI allows a baseline correction to be made, this is done by taking an average of the absorbances over a range of wavelengths at a flat part of the spectra and using this value for correction of the baseline. The causes of baseline drift included thermal effects on alignment and LED luminosity; since LED emission varies with junction temperature, a monitoring photodiode feeds reference inten- sity to the Pi which can then adjust LED current to stabilise source intensity. The GUI allows plotting of the spectra as well as calibration graphs with lines of best fit. Specific samples can be removed from the data and re-measured and the data replotted. The use of CEA spectrometer for single pass measurements allows determination of the molar extinction coefficient from a calibration graph of absorbance against standard analyte concentrations and this value is stored with the GUI and can be loaded into calculations for the CEA measurements for determining the figures of merit.

The CEF value can be used to calculate the effective pathlength of measurement $\left(l_{\mathrm{eff}}=l \times \mathrm{CEF}\right)$ where $l$ is the base pathlength. The effective pathlength is used in the calculation of the sensitivity of the measurement which is defined in terms of the minimum detectable change in the absorption coefficient $\alpha_{\min }$ (also known as noise-equivalent absorption coefficient). This is given by the expression:

$$
\alpha_{\min }=\frac{2.303 \Delta \mathrm{ABS}_{\min }}{l_{\text {eff }}}
$$

The value of $\alpha_{\min }$ as seen in eqn (9) can also be used to calculate the limit of detection (LOD) of an analyte which is defined as the smallest amount that can be reliably measured.

$$
\mathrm{LOD}=\frac{3 \alpha_{\min }}{2.303 \varepsilon_{\lambda}}
$$

The performance of the developed CEA spectrometer was determined using Rhodamine $\mathrm{B}$ as an analyte, giving a broad absorbance spectra with a peak wavelength of $\sim 554 \mathrm{~nm}$ (Fig. 2A). This wavelength was used to calculate all the figures of merit with measurements made over a concentration range of $2.08 \times 10^{-7}$ to $2.08 \times 10^{-6} \mathrm{M}$. Fig. $2 \mathrm{~B}$ shows the associated calibration graph of the cavity absorption versus concentration for Rhodamine B. It can be seen from Fig. 2B that the gradient of the cavity absorption is $1.25 \times 10^{7}$. This along with a calculated value for the molar extinction coefficient $\varepsilon$ of $93879 \mathrm{M}^{-1}$ $\mathrm{cm}^{-1}$ and pathlength of $1 \mathrm{~cm}$, from single pass measurement of absorbance versus concentration for Rhodamine B, was substituted into eqn (5) to give a CEF of 57.8. The effective pathlength is therefore also $57.8 \mathrm{~cm}$. Measurements from the CEA spectrometer gave a value of $1.28 \times 10^{-4}$ for $\Delta \mathrm{ABS}_{\min }(t)$ from eqn (7) with a one second acquisition time. It follows then from eqn (8) that $\alpha_{\text {min }}$ the sensitivity will be $5.1 \times 10^{-6} \mathrm{~cm}^{-1}$ and from eqn (9) and an LOD of $7.1 \times 10^{-11} \mathrm{M}$. It can be seen from Table 1 that the developed portable low cost CEA spectro-

\begin{tabular}{|c|c|c|c|c|c|c|}
\hline Study & Light source & Cell & $\lambda \mathrm{nm}^{-1}$ & $\alpha_{\min } / \mathrm{cm}^{-1}$ & LOD $\mathrm{M}^{-1}$ & $\varepsilon / \mathrm{M}^{-1} \mathrm{~cm}^{-1}$ \\
\hline Qu et al. ${ }^{19}$ & LED & Cuvette & 527 & $6.5 \times 10^{-5}$ & $8.5 \times 10^{-10}$ & $9.1 \times 10^{5}$ \\
\hline Seetohul et al. ${ }^{13}$ & LED & HPLC cell & 556 & $1.9 \times 10^{-5}$ & $2.1 \times 10^{-9}$ & $1.1 \times 10^{5}$ \\
\hline Fiedler et al. ${ }^{20}$ & LAMP & Cuvette & 607 & $2.0 \times 10^{-5}$ & - & - \\
\hline This study & LED & Cuvette & 554 & $5.1 \times 10^{-6}$ & $7.1 \times 10^{-11}$ & $9.4 \times 10^{4}$ \\
\hline
\end{tabular}
meter offers the highest sensitivities of comparable CEA

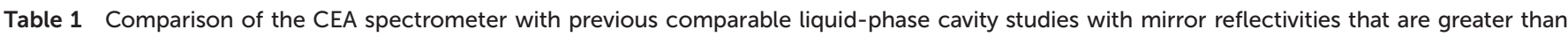
0.99 and a $1 \mathrm{~cm}$ pathlength giving key figures of merit of the $\alpha_{\min }$ LOD and the molar extinction coefficient for the specific analyte 
Table 2 Comparison of costs for key functional elements for earlier CEA ELISA study, ${ }^{17}$ CEA spectrometer and the CEA POC reader in this study

\begin{tabular}{|c|c|c|c|c|c|c|}
\hline Study & Light source & $\begin{array}{l}\text { Light } \\
\text { collim-ation }\end{array}$ & $\begin{array}{l}\text { Dielectric } \\
\text { mirrrors }\end{array}$ & Sample movement & Detector & $\begin{array}{l}\text { Overall } \\
\text { cost }\end{array}$ \\
\hline Seetohul et al., ${ }^{17}$ & PCB white LED $<£ 50$ & Fibre optic cable $£ 300$ & $99 \pm 0.5 \% £ 640$ & $\begin{array}{l}\text { Thorlabs XY scanning } \\
\text { stage \& controller } £ 8100\end{array}$ & $\begin{array}{l}\text { Andor spectrograph \& CCD } \\
\text { camera } £ 20500\end{array}$ & $\sim £ 29,600$ \\
\hline $\begin{array}{l}\text { This study CEA } \\
\text { spectrometer }\end{array}$ & PCB White LED $<£ 50$ & Fibre optic $£ 300$ & $99 \pm 0.5 \% £ 640$ & $\begin{array}{l}\text { Miniature linear rail \& } \\
\text { carriage } £ 250\end{array}$ & $\begin{array}{l}\text { Ocean optics } \\
\text { microspectrometer } £ 1100\end{array}$ & $\sim £ 2,300$ \\
\hline $\begin{array}{l}\text { This study CEA } \\
\text { POC }\end{array}$ & $\begin{array}{l}\text { PCB narrow band } \\
\text { LEDs }<£ 50\end{array}$ & - & $99 \pm 0.5 \% £ 640$ & $\begin{array}{l}\text { Rail assembly and motor } \\
\text { stand } £ 250\end{array}$ & Photodiode PCB $<£ 50$ & $\sim £ 1,000$ \\
\hline
\end{tabular}

studies and will have significantly higher sensitivity than conventional single pass absorbance instruments. We can also envisage further increases in the sensitivity of the CEA spectrometer by improving the approach for mirror alignment e.g. by use of a metal sample holder to provide more rigidity in place of the i3DP polymer holder.

\section{CEA point-of-care (POC) reader}

Point-of-care (POC) diagnostics are applicable for a variety of situations including within primary, secondary and tertiary care as well as within low resource and austere settings. They are, however, required to be low cost as well as being easy to use. As a consequence, CEA detection for POC diagnostics would require a significant further reduction in size and cost from that which was achieved for the CEA spectrometer. Colorimetric ELISAs are normally performed at key selected wavelengths, typically $450 \mathrm{~nm}$ and $540 \mathrm{~nm}$, and so cost and size reduction could firstly be achieved by replacing the microspectrometer with photodiodes. A range of optical arrangements were investigated for the CEA POC reader by simulation using Zeemax OpticStudio. This is a powerful optical simulation program that carries out ray-tracing of a light path as it passes through optical elements, such as lenses. This simulation work significantly reduces the time to optimise complex optical systems, and generally reduces the number of prototyping stages required.

The arrangement for the CEA spectrometer was used as the basis for simulation study with a key objective to maximise the light into the optical cavity to compensate for the losses from the high reflectivity mirrors. The simulation study showed that light from an optical fibre reduces signal strength (ESI, Fig. S1 $\dagger$ ) and there could therefore be benefit in removing this. A collimated beam from a high power LED was shown to give a factor of $10^{3}$ increase at the centre of the optical cavity, $c f .6 .28$ $\times 10^{-3}$ to $1.91 \times 10^{-6}$ lumens. To further maximise signal strength, the broadband light source was replaced with a narrowband light source, this reduced cost and increased light power through the sample, to further increase the sensitivity of the system. Table 2 shows a comparison of the costs of the main functional elements for an earlier CEA study for ELISA, ${ }^{17}$ the CEA spectrometer and the CEA POC reader. The costs do not include the mechanical, structural and other component elements or take into account the volumes manufactured and is a simple indication of the cost reduction that can be achieved. Table 2 shows that a near 30 -fold reduction in cost can be achieved - with the main cost reduction being achieved through the choice of the detector, sample movement and light collimation - so that that CEA detection is amenable for POC applications.

The optical arrangement for the CEA POC reader device (Fig. 3) was based on the simulation results. For the optical reading head (Fig. 3C and inset), a cube beam splitter allowed $50 \%$ of the LED power to pass through the sample, whilst the remaining $50 \%$ of the light from each LED passes out of the system. This remaining $50 \%$ of light from each LED is measured by a second photodiode, this being a reference beam. The purpose of the second photodiode is to monitor the light output fluctuation in the LEDs, and can be used for compensation. For example, if the LEDs begin to heat during operation, this may result in fluctuations in LED intensity (radiant flux). Without compensation, this drop in light intensity could be mistaken for an increase in absorption of light through the sample. This may yield erroneous results; as such the system has a reference photodiode which monitors the light output from the LEDs. To combat heat and undesirable light intensity changes, each LED is pulsed for a short duration to manage thermal effects associated with high powered LEDs. The "on time" of the LEDs has been optimised to ensure good thermal management whilst maintaining a good level of light passing through the sample.

A custom microwell plate with seven wells holding the assay is loaded onto a heated cradle (tray) within the CEA POC reader. The cradle has a heater element that regulates the sample temperature to $24^{\circ} \mathrm{C} \pm 1^{\circ} \mathrm{C}$. Once the microwell plate is loaded, the CEA POC reader moves the cradle and microwell plate with assay in line with the optical reading head. The system was designed to take a reading from each well, in sequence one well at a time, and to be able to make multiple readings of each well every few seconds. This can be modified depending on the desired sample rate. The microwell plate used glass slide for both the bottom and top layers to maximise the light power (signal). Alternative polystyrene (PS) material was also tested but this was found to significantly reduce the signal strength due to scattering which arises from a less smooth surface. The CEA POC reader was prototyped using a combination of 3D printed and machined aluminium parts with some custom electronics and off-the-shelf development kits.

\section{Sepsis POC diagnostic with CEA detection}

Sepsis is a life-threatening illness caused by a systemic reaction to an infection - usually bacterial but also viral or fungal. 
Cytokines released into the blood, in response to infection, can induce widespread inflammation which leads to abnormal cardiovascular response and damage to the body's organs. In the worst cases the patient can develop septic shock with multi-organ failure. Sepsis remains the primary cause of death from infection with around 6 million deaths annually and with the majority of these preventable. ${ }^{21}$ Sepsis is a growing problem for a number of reasons including: aging populations; more patients living with chronic disease; and the evolution of more drug-resistant pathogens. Sepsis can arise unpredictably and progress rapidly, every hour that sepsis is undiagnosed increases mortality by $6-10 \%{ }^{22}$ and so early diagnosis and prompt action is vitally important to reduce mortality and cost of treating patients. Sepsis is difficult to diagnose in the early stages and so a low cost POC diagnostic that could provide clinicians with early indication of biomarkers for sepsis would be beneficial.

There are a very large number of biomarkers that have been studied for sepsis and we have previously reviewed these. ${ }^{23}$ We have focussed here on CRP, PCT and IL-6 as amongst the most widely studied biomarkers for the diagnosis of sepsis. PCT and CRP are produced in response to infection and/or inflammation and are the most widely used clinical biomarkers, apart from lactate, for the diagnosis and management of patients with sepsis. CRP has long been used to indicate presence of significant inflammatory and infectious disease because its levels rise more significantly than other acute phase reactants. The main drawback for CRP is that it has low specificity as a biomarker for sepsis within adults. It is, however, commonly used to screen early onset sepsis (occurring during the first 24 hours of life) because it has high sensitivity in this setting. PCT is produced within parenchymal tissues in response to bacterial infection and could have a use within antimicrobial stewardship. IL-6 has an important role in the inflammatory response leading to production of CRP by the liver as well as influencing B- and T-cell activity. No one biomarker is likely to adequately reflect the rapidly changing nature of a potentially septic patient's status, a better approach would be to provide clinicians with information for a panel of biomarkers.

The CEA POC reader was compared with the EL800 (Biotek) and LT-4000 (EuroClone) microplate readers using Tartrazine dye as a standard. The CEA POC reader was able to measure to $1.2 \times 10^{-7} \mathrm{M}$, albeit with a higher coefficient of variation, whilst the EL800 and LT-400 were only able to measure to respectively $1.2 \times 10^{-6} \mathrm{M}$ and $2.3 \times 10^{-6} \mathrm{M}$. Moreover, a larger slope of signal against concentration would suggest higher sensitivity with greater resolution between measurements. Fig. 4A shows the multi-pass absorption against Tartrazine concentration for the CEA POC reader and in Fig. 4B the single pass absorbance against Tartrazine concentration for the EL800 and LT-4000 microplate readers. The CEF should ideally be gained through single and multiple pass measurements with the CEA POC reader and using eqn (5). The CEA POC reader, however, has a simple fixed optical arrangement that only allows sensitive multiple pass measurements to be made. The CEF can be approximated by using the gradient of multipass measurements from the CEA POC reader, Fig. 4A, and single pass measurements, Fig. $4 \mathrm{~B}$, or alternatively by substituting a value for the molar extinction coefficient $(\varepsilon)$ of Tartrazine within eqn (5). Using the gradients of the multipass and single pass measurements from Fig. 5 gives a CEF of 14. The low cost and portable CEA POC reader therefore offers higher sensitivity than existing single pass microplate readers. The CEF for the CEA POC reader was, however, lower than for the CEA spectrometer but this could be expected since the former uses a low cost photodiode rather than a microspectrometer for detection. Moreover, the CEA POC reader used

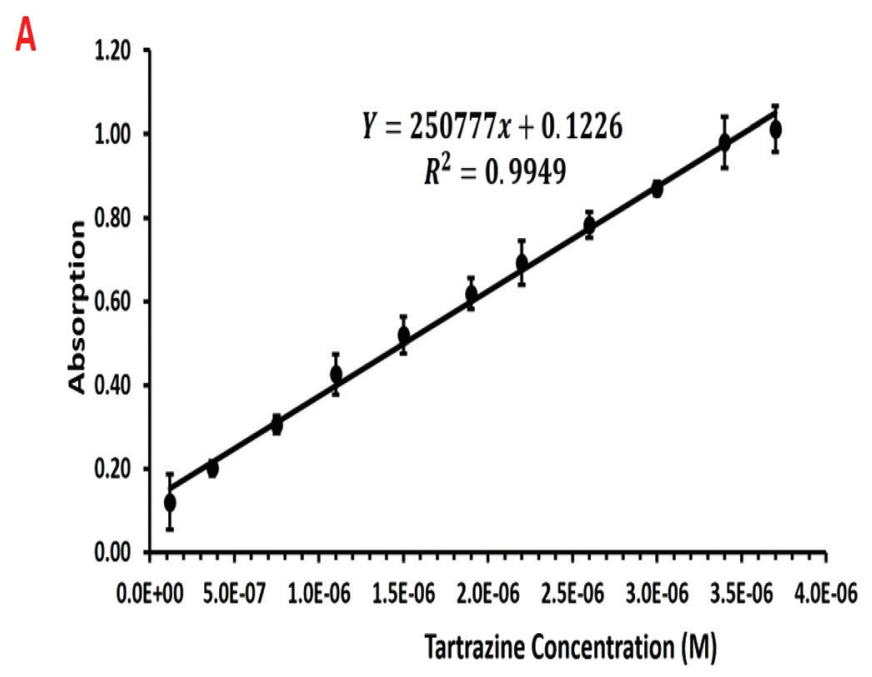

B

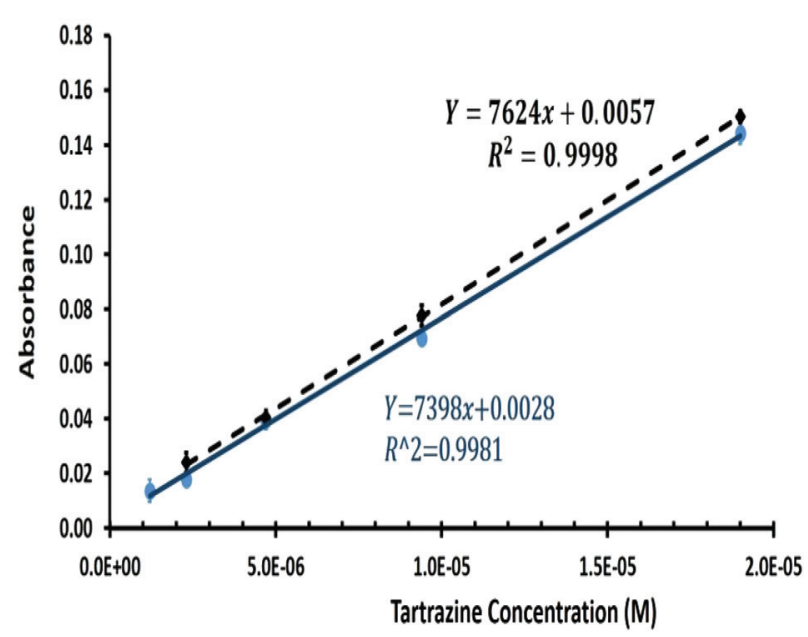

Fig. 4 (a) Multi-pass absorption versus tartrazine concentration using the CEA POC reader and (b) conventional single pass absorbance versus tartrazine concentration using the el 800 (dark blue line) and LT-4000 (dashed black line) microplate readers. the confidence limits are shown for four replicate measurements at $95 \%$. the CEA POC reader was able to measure to $1.2 \times 10^{-7} \mathrm{~m}$ compared with el 800 to $1.2 \times 10^{-6} \mathrm{~m}$ and LT-400 to $2.3 \times$ $10^{-6} \mathrm{~m}$, so an order of magnitude higher sensitivity compared with the more sensitive microplate reader. 
A

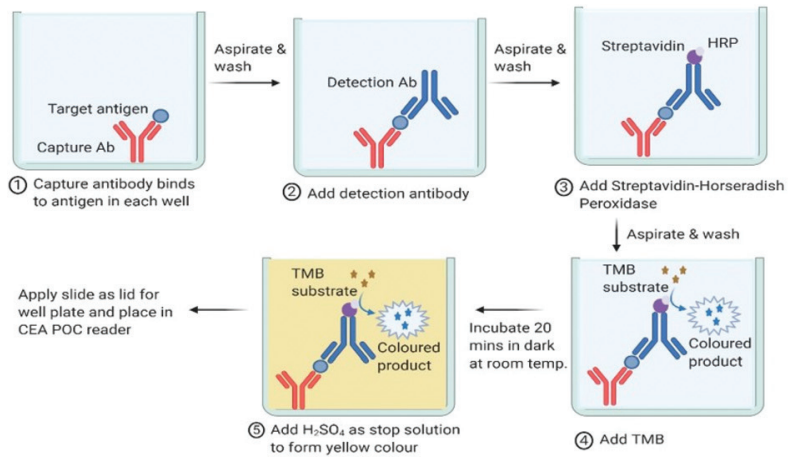

C

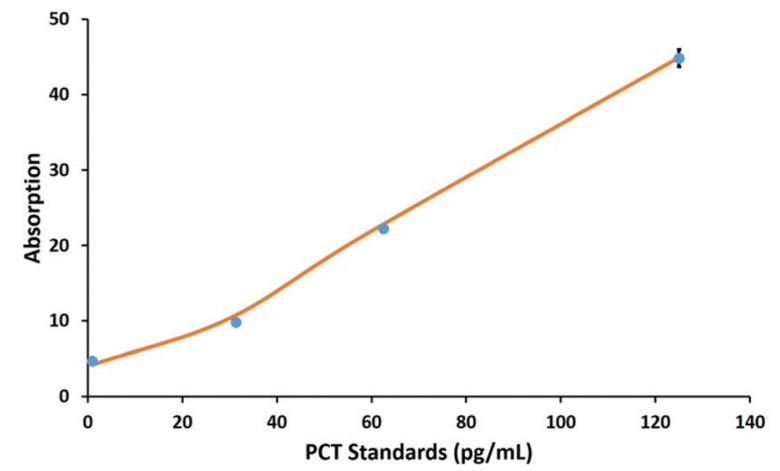

B

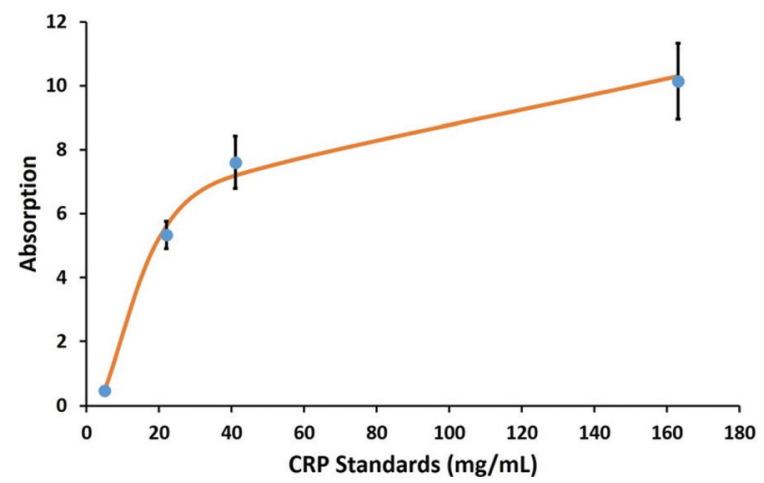

D

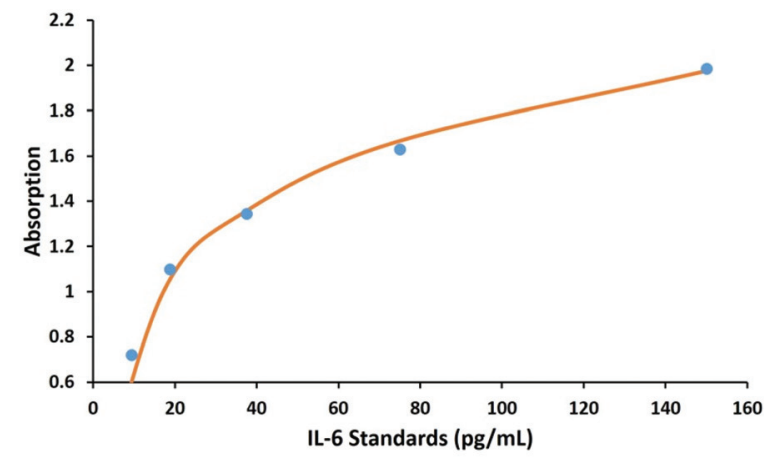

Fig. 5 (a) Immobilisation and assay approach for the sepsis biomarkers in microwell plate. standard elisa curves for (b) CRP (c) PCT and (d) IL-6 with each data point as the mean of triplicate measurements.

10-bit resolution for light detection. At very low concentrations, with low light absorption, there may be some saturation of the detector. This could potentially be improved by implementing 20-bit resolution and lock-in detection for the light sensing.

The CEA POC reader has been demonstrated for CRP, PCT and IL-6 - towards a three biomarker panel for sepsis - using enzyme linked immunosorbent assay (ELISA) within five wells of the microwell plate. Fig. 5A shows a generalised immobilisation and assay procedure for the sepsis biomarkers. A glass slide is place on top of the microwell plate to form the lid and this is then placed within the CEA POC reader. The intensity of the colour product formed in the reaction was measured by the CEA POC reader at $450 \mathrm{~nm}$ and $540 \mathrm{~nm}$. Calibration standards were used to construct standard curves, using four parameter logistic curve fitting, for the three sepsis biomarkers and these are shown in Fig. 5b-d. Although assay for CRP is readily available within secondary care settings (hospitals) this is not as widely true for PCT and IL-6. Information about the concentrations of the three biomarkers would give a clinicians considerable information about the patient status for decision making. It should also be noted that immunoassay can be implemented in a wide variety of formats so that optical cavity approaches could have a wide variety of applications. ${ }^{24}$

The microwell plate structure was to demonstrate applicability of the CEA POC Reader for immunoassay. There was some variability in the measurements made and this could have arisen for a number of reasons. There is a need to place a glass slide as a lid on the microwell plate to provide a flat interface for the optical path. High interface scattering losses occur where a well is not filled to capacity and has a concave shaped meniscus. Scattering losses are reduced by placing a glass slide on the microwell plate as a lid to provide a planar surface but this could lead to some imprecision since some fluid may spill from one well to another leading to contamination of sample. Moreover, some bubbles were observed within the wells which would interfere with the optical beam and lead to errors. A key issue of CEA detection within liquids and for immunoassay are the scattering losses that may arise. The volume of wells within the microwell plate is less than half that of a 96-well standard microplate and decreasing the volume further as well as ensuring no particles and bubbles could decrease the scattering losses and increase the sensitivity and precision. It should, however, be noted that decreasing the pathlength will also impact on the sensitivity. A higher level of precision could therefore be achieved by incorporating the immunoassay within a microwell based microfluidic device where the immunoassay and washing operations are automated using fluid driving. We have previously described a pressurised fluid driving system with flow rates controlled down to $0.7 \mu \mathrm{L} \mathrm{min}{ }^{-1}$ and with automated fluid switching using a manifold controlled by solenoid valves. ${ }^{25}$

The cost, size and simplicity of CEA detection make this amenable for portable or point-of-care use. In some instances compromises will need to be made in terms of choice of functional components and performance but depending on the 
application and the configuration used then this need not always be the case. A polymer microfluidic device that incorporates the immunoassay for the selected sepsis biomarkers could be produced in volume at low cost using manufacturing approaches such as microinjection moulding. The key requirement would be that the surface of the microfluidic device within the light path or optical cavity - is very smooth to avoid scattering and increase the signal strength. A smooth polymer based microfluidic could be achieved using custom moulding with a high optical quality injection moulding tooling. Further improvements could also include the alignment of the mirror system (optical cavity), and the precision of the electromechanical system for moving the consumable between wells. With more sophisticated manufacturing processes available at commercial product volumes, it is expected that many of these variables could be understood, controlled or potentially eliminated, resulting in more consistency at very low concentrations.

Fluorescence is typically regarded as being 100 to 1000 times more sensitive than absorbance measurements. CEA detection can offer practically, within liquids, a 10 to 100 times higher sensitivity than conventional absorbance measurements so that it is more comparable to fluorescence measurements without the drawback of having to use expensive fluorophore labels. It would also allow existing colorimetric labels to be used with higher sensitivity or, given the large number of molecules that absorb in the UV and visible region, use within assays where there is no requirement for a label. The CEA POC reader could include WiFi or Blutetooth connectivity to enable Cloud-based information sharing between primary (General Practitioner office) or tertiary care (care homes) or low resource settings to a central secondary care site. ${ }^{26}$ A low cost microfluidic element that incorporates an assay with CEA POC reader offering highly sensitive absorption measurements could be applicable in a variety of clinical contexts. Such applications could include low cost point-ofcare diagnosis of COVID-19 by detection of SARS-CoV-2 using a colorimetric loop-mediated isothermal amplification (LAMP) assay within a microfluidic device.

\section{Conclusion}

We have reported here a small portable and robust CEA spectrometer as a broad spectrum device which uses fibre optic coupled spectrometer and fibre optic couple white light source. The CEA spectrometer was shown to have a cavity enhancement factor of 57.8 and an $\alpha_{\text {min }}$ of $5.1 \times 10^{-6} \mathrm{~cm}^{-1}$ which is lower than that reported in other studies in the literature and at a lower cost. We have also demonstrated further significant cost reduction for a CEA POC reader through use of a photodiode in place of microspectrometer, to target wavelengths of interests rather than a broader spectrum. Moreover, the costs are also reduced and the light (power) through the sample increased by removing the fibre optic elements and use of narrow band (single wavelength) LED. The CEA POC reader was demonstrated for determination of CRP, PCT and
IL-6, within the relevant clinical range, towards a three biomarker panel for diagnosis of sepsis. Our future work will focus on improving the precision of the signal response particularly at the very low concentration range. This can be done by use of a microfluidic device for automation of the immunoassay as well as optimising the cavity arrangement. We will also seek to further reduce the cost of the CEA POC reader by simplifying the optical components and designing for volume production.

\section{Conflicts of interest}

The authors declare no conflict of interest. This paper does not raise any ethical issues.

\section{Acknowledgements}

This report contains independent research funded by the National Institute for Health Research under its Invention for Innovation (i4i) Programme (Grant reference number II-BP-0817-10012 Sepsis Point-of-Care Diagnostic System using Cavity Enhanced Absorption (SepsisPoC). The views expressed in this publication are those of the authors and not necessarily those of the National Institute for Health Research or the Department of Health and Social Care. This work was also supported by the European Commission through the Horizon 2020 GateOne project (project number 644856). Z. Ali has financial interest in Anasyst Ltd which has licensed CEA technology from Teesside University. We wish to thank and remember the many valuable contributions of $\mathrm{Dr}$ Simon M. Scott who sadly passed away before preparation of this work.

\section{References}

1 J. E. Thompson, J. Spectrosc., 2017, 2017, 1-10.

2 C. M. Rushworth, J. Davies, J. T. Cabral, P. R. Dolan, J. M. Smith and C. Vallance, Chem. Phys. Lett., 2012, 554, 114.

3 A. O'Keefe and D. A. G. Deacon, Rev. Sci. Instrum., 1988, 59, 2544-2551.

4 D. James, B. Oag, C. M. Rushworth, J. W. L. Lee, J. Davies, J. T. Cabral and C. Vallance, RSC Adv., 2012, 2, 5376-5384.

5 S.-S. Kiwanuka, T. K. Laurila, J. H. Frank, A. Esposito, K. Blomberg von der Geest, L. Pancheri, D. Stoppa and C. F. Kaminski, Anal. Chem., 2012, 84, 5489-5493.

6 U. Elejalde and J. M. Girkin, Appl. Opt., 2007, 46, 3995.

7 R. Engeln, G. Berden, R. Peeters and G. Meijer, Rev. Sci. Instrum., 1998, 69, 3763-3769.

8 S. E. Fiedler, G. Hoheisel, A. A. Ruth and A. Hese, Chem. Phys. Lett., 2003, 382, 447-453.

9 S. M. Ball, J. M. Langridge and R. L. Jones, Chem. Phys. Lett., 2004, 398, 68-74. 
10 L. van der Sneppen, G. Hancock, C. Kaminski, T. Laurila, S. R. Mackenzie, S. R. T. Neil, R. Peverall, G. A. D. Ritchie, M. Schnippering and P. R. Unwin, Analyst, 2010, 135, 133139.

11 S.-S. Kiwanuka, T. Laurila and C. F. Kaminski, Anal. Chem., 2010, 82, 7498-7501.

12 M. Islam, L. N. Seetohul and Z. Ali, Appl. Spectrosc., 2007, 61, 649-658.

13 L. N. Seetohul, Z. Ali and M. Islam, Anal. Chem., 2009, 81, 4106-4112.

14 Z. Bajuszova, H. Naif, Z. Ali, J. McGinnis and M. Islam, Analyst, 2018, 143, 493-502.

15 S. R. T. Neil, C. M. Rushworth, C. Vallance and S. R. MacKenzie, Lab Chip, 2011, 11, 3953-3955.

16 C. M. Rushworth, G. Jones, M. Fischlechner, E. Walton and H. Morgan, Lab Chip, 2015, 15, 711-717.

17 Z. Bajuszova, Z. Ali, S. Scott, L. N. Seetohul and M. Islam, Anal. Chem., 2016, 88, 5264-5270.

18 M. Mazurenka, A. J. Orr-Ewing, R. Peverall and G. A. D. Ritchie, Annu. Rep. Prog. Chem., Sect. C: Phys. Chem., 2005, 101, 100.
19 Z. Qu, J. Engstrom, D. Wong, M. Islam and C. F. Kaminski, Analyst, 2013, 138, 6372.

20 S. E. Fiedler, A. Hese and A. A. Ruth, Rev. Sci. Instrum., 2005, 76, 023107.

21 K. Reinhart, R. Daniels, N. Kissoon, F. R. Machado, R. D. Schachter and S. Finfer, N. Engl. J. Med., 2017, 377, 414-417.

22 A. Kumar, D. Roberts, K. E. Wood, B. Light, J. E. Parrillo, S. Sharma, R. Suppes, D. Feinstein, S. Zanotti, L. Taiberg, D. Gurka, A. Kumar and M. Cheang, Crit. Care Med., 2006, 34, 1589-1596.

23 A. Teggert, H. Datta and Z. Ali, Micromachines, 2020, 11, 286.

24 S. Armstrong, Z.-F. Miao, F. J. Rowell and Z. Ali, Anal. Chim. Acta, 2001, 444, 79-86.

25 M. Parekh, A. Ali, Z. Ali, S. Bateson, F. Abugchem, L. Pybus and C. Lennon, Analyst, 2020, 6148-6161.

26 S. O’Sullivan, Z. Ali, X. Jiang, R. Abdolvand, M. S. Ünlü, H. P. Da Silva, J. T. Baca, B. Kim, S. Scott, M. I. Sajid, S. Moradian, H. Mansoorzare and A. Holzinger, Sensors, 2019, 19, 1-31. 